\title{
Neuroprotective role of human neuritin 1 on axotomized human eyes pressurized using the Translaminar Autonomous System
}

\author{
Ryan Miller ${ }^{1}$, Shahna Shahul Hameed ${ }^{1}$, Tasneem Sharma ${ }^{1}$ \\ ${ }^{1}$ Department of Ophthalmology, Indiana University School of Medicine, Indiana University - \\ Purdue University
}

Purpose: Primary open-angle glaucoma is a leading cause of vision loss worldwide characterized by retinal ganglion cell death (RGC) and visual field loss. Glaucoma is most commonly associated with an increase in intraocular pressure (IOP), and current clinical management is limited to lowering intraocular pressure. We have previously shown that the secreted human protein neuritin 1 (NRN1) exhibits neuroprotection, regeneration, and preservation of RGC function after RGC axonal injury. We propose to overcome RGC degeneration by over-expressing NRN1 in our novel ex-vivo perfused pressurized Translaminar Autonomous System (TAS).

Methods: Human donor eyes were obtained from eye banks. To model glaucomatous conditions, dissected human posterior eye cups were cultured in the TAS model with IOP:ICP conditions of $16: 12 \mathrm{mmHg}$ over 6 days. Flow rate of perfusion medium to the interior and exterior (around optic nerve) of the eye cup (IOP and ICP, respectively) was controlled by automated pumps to induce the translaminar pressure gradient.We assessed survival of RGCs with and without hNRN1 treatment by TaqMan array analysis of apoptosis/inflammatory markers (retina). Expression of FN and COLIV was examined from conditioned medium of both groups by Western analysis.

Results: We successfully maintained IOP: ICP differentials over 6 days. The RGCs degenerated faster in non-treated conditions compared to NRN1 treated eyes. In contrast to control, NRN1treated group displayed differential expression of FN and COLIV. We observed dysregulated expression of GFAP, BAX and TLR4 expression between control and experimental conditions.

Conclusion: We utilized our unique TAS model to recreate the human optic nerve head environment of elevated translaminar pressure for testing a unique neuroprotective and regenerative gene therapy. Using this approach, we hope to identify human Neuritin 1 as a therapeutic gene to save the visual neurons and develop a new treatment for glaucoma. 